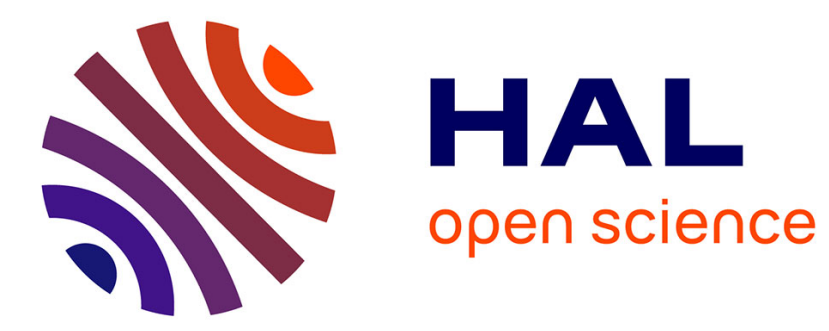

\title{
Phase diagram of quasi-one-dimensional conductors in strong magnetic field
}

M. Héritier, G. Montambaux, P. Lederer

\section{To cite this version:}

M. Héritier, G. Montambaux, P. Lederer. Phase diagram of quasi-one-dimensional conductors in strong magnetic field. Journal de Physique Lettres, 1985, 46 (17), pp.831-836. 10.1051/jphyslet:019850046017083100 . jpa-00232906

\section{HAL Id: jpa-00232906 https://hal.science/jpa-00232906}

Submitted on 1 Jan 1985

HAL is a multi-disciplinary open access archive for the deposit and dissemination of scientific research documents, whether they are published or not. The documents may come from teaching and research institutions in France or abroad, or from public or private research centers.
L'archive ouverte pluridisciplinaire HAL, est destinée au dépôt et à la diffusion de documents scientifiques de niveau recherche, publiés ou non, émanant des établissements d'enseignement et de recherche français ou étrangers, des laboratoires publics ou privés. 
Classification

Physics Abstracts

$71.25-72.15-72.80-75.10$

\title{
Phase diagram of quasi-one-dimensional conductors in strong magnetic field
}

\author{
M. Héritier, G. Montambaux and P. Lederer \\ Physique des Solides, Bât. 510, Université Paris Sud, Centre d’Orsay, 91405 Orsay Cedex, France
}

(Reçu le 3 mai 1985, accepté sous forme définitive le 8 juillet 1985)

\begin{abstract}
Résumé. - Nous dérivons le diagramme de phase des conducteurs quasi-1D en champ magnétique. Nous réalisons un calcul microscopique des coefficients de Ginzburg-Landau de l'énergie libre. Ceuxci montrent un comportement original en fonction du champ. En conséquence, il apparaît, en champ croissant différents régimes d'ordre Onde de Densité de Spin, correspondant à différents types de « nesting » quantifié. Cette description rend compte de presque toutes les données expérimentales sur (TMTSF) ${ }_{2} \mathrm{ClO}_{4}$.
\end{abstract}

\begin{abstract}
The phase diagram of quasi-1D conductors in magnetic field is derived. We perform a microscopic calculation of the Ginzburg-Landau free energy coefficients. They exhibit a novel behaviour as a function of field. As a result, different regimes of SDW ordering appear with increasing field, corresponding to different types of quantized nesting. This description may account for nearly all the available experimental data in (TMTSF) ${ }_{2} \mathrm{ClO}_{4}$.
\end{abstract}

The organic conductors of the (TMTSF) ${ }_{2} \mathrm{X}$ family [1] are strongly anisotropic crystalline systems, with weakly coupled one-dimensional stacks of nearly planar TMTSF organic molecules, separated by monovalent inorganic anions such as $\mathrm{PF}_{6}^{-}, \mathrm{ClO}_{4}^{-}$, etc... They exhibit a large variety of phase transitions for moderate variations of external parameters, such as temperature, pressure, magnetic field, etc..., revealing a competition between different ground states of neighbouring energies. At room temperature, these salts are metallic, but the ground states vary from metal to superconductor, Spin Density Wave (SDW) etc..., depending on the particular anion and the pressure [1].

The semimetallic [2-8] SDW [9, 10] phases stabilized when a magnetic field is applied in the $c^{*}$-direction to metallic (TMTSF) ${ }_{2} \mathrm{PF}_{6}$ or (TMTSF) ${ }_{2} \mathrm{ClO}_{4}$ exhibit novel properties. Gor'kov and Lebed [11] have explained the destabilization of the metallic open Fermi surface in a moderate magnetic field by an orbital effect [12-14], which makes the electron motion more one-dimensional. Magnetoresistance [2, 4], Hall effect [5, 8] and specific heat [15] experiments above the threshold field have proved the occurrence of a cascade of Field-Induced SDW phase transitions. Within each FISDW, a Hall plateau develops at low $T$ [7, 8]. The cascade of transitions has been interpreted as a quantized nesting effect [12] : the nesting wave vector adapts to the field so that the ratio of the semimetallic Fermi surface area to the area quantum is an integer [12]. Within each phase, the SDW pairing provides a new mechanism for the realization of a Quantized Hall effect [7, 8, 12]. 
These experimental data suggest new possibilities and give rise to more questions : can we obtain the equivalent of the Laughlin's state [16] for fractional filling of the last Landau level in stronger field ? How is it affected by the Fermi surface anisotropy ? Can the Quantized Hall Effect be influenced by the periodic lattice potential [17] in fields available in the laboratory, as suggested by the anion ordering effect in (TMTSF) ${ }_{2} \mathrm{ClO}_{4}[8]$ ?

The answers to these questions require a detailed understanding of the phase diagram, in particular a description of the ordered phase. There is a general agreement on the fact that the phenomenon is an orbital effect on anisotropic three-dimensional Fermi surfaces [6-8, 11-15, 18] and not a one-dimensional fluctuation effect [19]. However, the detailed interpretations [7-14] diverge, and none of them seems to explain the experimental data in the whole field range, although some [12] may account for the moderate field $(H<7 \mathrm{~T})$ experiments $[4,5,7,8,15]$ and others $[11,13,14,20]$ for the strong field ones $(H>7 \mathrm{~T})[6,21,22]$.

Solving these contradictions is essential to the understanding of the interplay between quantum orbital effect and phase transitions. The purpose of this Letter is to give a consistent qualitative picture of the entire phase diagram. We reconcile the various interpretations in a theoretical description which seems to account for nearly all the available experimental data through a microscopic Ginzburg-Landau description of the ordered phase.

The condition of formation of a SDW phase, discussed in the three-dimensional regime in the Fermi liquid theory, is given by Stoner's criterion $1-\lambda \chi^{0}(\mathbf{Q}, H, T)<0$, where $\lambda$ is the molecular field constant and $\chi^{0}(\mathbf{Q}, H, T)$ the magnetic susceptibility of non interacting electrons at wave vector $\mathbf{Q}$. The metallic Fermi surface is described in a simple orthorhombic model. In a zeroth order approximation, the dispersion relation of electron with wave vector $\mathbf{k}$ is given by :

$$
\begin{aligned}
& \varepsilon^{0}(\mathbf{k})= \pm v\left(k_{x} \pm k_{\mathrm{F}}\right)+\varepsilon_{\perp}^{0}\left(\mathbf{k}_{\perp}\right) \\
& \varepsilon_{\perp}^{0}\left(\mathbf{k}_{\perp}\right)=-2 t_{b} \cos k_{y} b-2 t_{c} \cos k_{z} c
\end{aligned}
$$

such a Fermi surface would be completely unstable $v$ s. formation of a SDW at wave vector $\mathbf{Q}_{0}=\left(2 k_{\mathrm{F}}, \frac{\pi}{b}, \frac{\pi}{c}\right)$ because of the perfect nesting [18]. Small (although essential for the stability of the metallic phase) second harmonics in $\varepsilon(\mathbf{k})$ must be considered :

$$
\varepsilon_{\perp}(\mathbf{k})=\varepsilon_{\perp}^{0}(\mathbf{k})-2 t_{b}^{\prime} \cos 2 k_{y} b-2 t_{c}^{\prime} \cos 2 k_{z} c .
$$

Their main origin is deviation from linearity of the longitudinal dispersion relation and the existence of several important transfer integrals in the transverse direction [23]. Typical values of the various terms are $t_{b} \sim 300 \mathrm{~K}, t_{c} \sim 10 \mathrm{~K}, t_{b}^{\prime} \sim 10 \mathrm{~K}, t_{c}^{\prime} \sim 0.3 \mathrm{~K}$. A more realistic band structure would modify details of the calculation, in particular the exact best nesting wave vector [23], but would not affect qualitatively the theory.

First, we make the assumption that $\lambda$ is independent of the wave vector. The SDW wave vector yielding the highest critical temperature is close to $\mathbf{Q}_{0}$, but depends on the magnetic field [12] : $\mathbf{Q}_{\mathrm{T}}(H)=\left(2 k_{\mathrm{F}}+q_{x}, \frac{\pi}{b}+q_{y}, \frac{\pi}{c}\right)$, where $q_{x}(H)=n e H b / c$ is quantized ( $n$ is an integer). Although this condition yields very small modulations of $\mathbf{Q}_{T}$, which would seem to be destroyed by a small amount of disorder, we have checked that the experimental mean free path in (TMTSF) ${ }_{2} X$ compounds in large enough to have no effect on the quantization condition $[5,8]$. Each integer value of the quantum number $n$ labels a different SDW phase. The corresponding critical temperatures $T_{n}$ are given, within logarithmic accuracy, by :

$$
\operatorname{Ln}\left(\frac{2 \pi c T_{n}}{e H v b}\right)=\frac{\operatorname{Ln}\left(\pi T_{0} / 4 t_{b}^{\prime}\right)}{J_{n}\left(2 c t_{b}^{\prime} / e H v b\right)}
$$


(Numerical evaluation of $T_{n}$ yields significant corrections to equation(2); our point in this Letter is a qualitative one, so we disregard them here.) $T_{0}$ is the SDW critical temperature corresponding to dispersion relation (1) in zero field. It is related to $\lambda$ by $\operatorname{Ln}\left(\pi T_{0} d / v_{F}\right)=-2 \pi v_{F} b c / \lambda$, where $d$ is the lower distance cut-off.

The validity of the theory along the critical line may be extended to the ordered phase, near enough to the critical line, by a Ginzburg-Landau expansion of the free energy density $F_{n}=F_{0}+a_{n} \Delta_{n}^{2}+b_{n} \Delta_{n}^{4} . F_{0}$ is the metallic phase free energy and $\Delta_{n}$ is the gap in the SDW phase defined by the quantum number $n$. Microscopic calculations of the coefficients $a_{n}$ and $b_{n}$ are derived from the diagrams given in figure 1 . One obtains the order parameter and the free energy of each phase :

$$
\begin{gathered}
\Delta_{n}=\frac{32 f_{n}(H) t_{b}^{\prime}\left(T_{n}-T\right)}{3\left(c_{1}+c_{2} f_{n}(H)+c_{3} f_{n}^{2}(H)\right)} \\
F_{n}-F_{0}=-\frac{2\left(T-T_{n}\right)^{2}}{3 v b c\left(c_{1}+c_{2} f_{n}(H)+c_{3} f_{n}^{2}(H)\right)}
\end{gathered}
$$

where $f_{n}(H)=T /\left(2 t_{b}^{\prime} J_{n}^{2}\left(2 t_{b}^{\prime} / e H v b\right)\right)$.

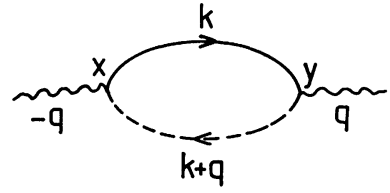

a)

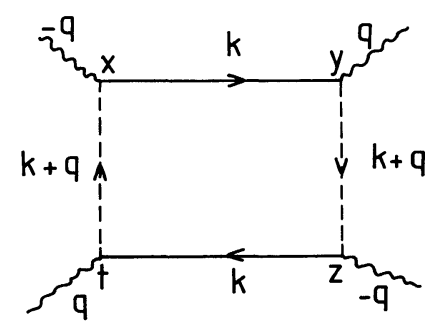

b)

Fig. 1. - (a) Diagram for the harmonic term in the free energy. The full lines refer to a particle on one side of the open Fermi surface; the dotted line to a particle on the other side. The wiggly lines represent the SDW order parameter. (b) Typical diagram for the anharmonic term in the free energy.

Orders of magnitude of the $c_{i}$ 's are : $c_{1} \sim 0.05, c_{2} \sim 1$ and $\left|c_{3}\right| \lesssim 1$ so that the term $c_{3} f_{n}(H)$ in the denominators may be neglected. From this expansion, one obtains with increasing field, a cascade a weakly first order transitions, corresponding to decreasing values of $n$. Because of the term $c_{2} f_{n}^{2}(H)$, the slopes of the transition lines from $n$ to $n-1$ are positive in the $(H, T)$ plane. The SDW wave vector oscillates with the field around the transverse best nesting wave vector in zero field. In this model, a value of $t_{b}^{\prime} \sim 10 \mathrm{~K}$ may account for the frequency of the transition cascade [4-8, 15] in the field range $3.8 \mathrm{~T}<H<7 \mathrm{~T}$ as well as for the number of carriers at low temperature $[7,8]$.

We also expect the occurrence of Hall plateaux [12]. In contrast with the standard Quantized Hall Effect, their heights may depend on temperature because of fluctuations of SDW phases corresponding to different values of $n$. In the low temperature limit, the Hall voltage should tend to temperature independent values $V_{n}=h / g n e^{2}$, where $g$ is a degeneracy factor, if we assume that our description remains valid at low $T$, and that magnetic breakdown does not occur. Such a behaviour seems to have been observed for the last three plateaux at fields lower than the $7.5 \mathrm{~T}$ strongly irreversible step [7, 8]. The heights of these three plateaux seem to extrapolate, at low $T$, to constant values which are in the ratio $\frac{1}{3}: \frac{1}{2}: 1[7,8]$. If this observation is confirmed, it might indicate (with the same caveat as above) that these plateaux correspond to the phases $n=3, n=2$ and $n=1$ respectively. However, if $\lambda$ is independent of $q$, the longitudinal nesting $\mathrm{Q}_{\mathrm{L}}=\left(2 k_{\mathrm{F}} 4 t_{\mathrm{b}} / v_{\mathrm{F}}\right.$, 
0,0 ) is always less stable than the transverse one (the critical temperature for $\mathbf{Q}_{L}$ is obtained from expression (3) by replacing $t_{b}^{\prime}$ by $t_{b}$ and $b$ by $b / 2$ ). In such a case, one cannot explain either the much more strongly first order transition observed at $7.5 \mathrm{~T}$ in Hall effect $[7,8]$ or at $9 \mathrm{~T}$ in magnetoresistance [4] (with probably differently relaxed samples) or the high frequency Shubnikov-de Haas oscillations observed in high fields $[5,6,21,22]$.

However, exchange interactions have probably a finite spatial range, so that in fact $\lambda$ and therefore $T_{0}$ depend on $\mathbf{Q}$. We assume that $\lambda$ favours the longitudinal wave vector $\mathbf{Q}_{\mathbf{L}}$. In such a case, the critical temperatures $T_{n}\left(\mathbf{Q}_{\mathrm{T}}\right)$ corresponding to the transverse wave vector is obtained from (2) by setting $T_{0}=T_{0}\left(Q_{T}\right)$, while those corresponding to $\mathbf{Q}_{\mathrm{L}}$ are given by

$$
\operatorname{Ln}\left(\frac{4 \pi c T_{n}}{e H v b}\right)=\frac{\operatorname{Ln}\left(\pi T_{0}\left(\mathbf{Q}_{\mathrm{L}}\right) / 4 t_{b}\right)}{J_{n}^{2}\left(4 c t_{b} / e H v b\right)}
$$

where $T_{0}\left(\mathbf{Q}_{\mathrm{L}}\right)>T_{0}\left(\mathbf{Q}_{\mathrm{T}}\right)$.

The critical lines $T_{n}(H)$ corresponding to the two wave vectors cross at a finite field, yielding a first order transition from $\mathbf{Q}_{\mathrm{T}}$ in low field to $\mathbf{Q}_{\mathrm{L}}$ in large field, with a discontinuous jump of the wave vector at the transition. For a reasonable $\lambda(\mathbf{Q})$, the transition is likely to occur for small transverse quantum number $n \sim 1$. This might explain the strongly hysteretic transition observed at 7.5-9 $\mathrm{T}[4,7,8]$. However, in this picture, the Hall voltage above the transition would be much smaller, because of much larger Fermi surface pocket. Therefore, we propose another longitudinal wave vector $\mathbf{Q}_{\mathrm{L}_{0}}(H)=\left(2 k_{\mathrm{F}}+\delta_{x}, \delta_{y}, 0\right)$ with $\delta_{x, y} \ll t_{b} / v_{\mathrm{F}}$, corresponding to almost compensated Fermi surface, as suggested in reference [14], assumed to be favoured by an interaction peaked at $\left(2 k_{\mathrm{F}}, 0,0\right)$ (S. Barišić has suggested the rôle of Umklapp scattering [24]). Maximizing the critical temperature corresponding to $\mathbf{Q}_{\mathbf{L}_{0}}$, one obtains a condition of quantization of $\delta_{x}(H)$ (and therefore of the albegraic number of carriers) : $\delta_{x}(H)=n e H b / c$. Then $\delta_{y}(H)$ is determined by maximizing $T_{\mathrm{c}}\left(\delta_{x}, \delta_{y}\right)=T_{n}\left(\delta_{y}\right)$ :

$$
\operatorname{Ln}\left(\frac{4 c T_{n}}{e H b v}\right)=\frac{\operatorname{Ln}\left(\pi T_{0}\left(\delta_{x}, \delta_{y}\right) / 4 t_{b}\right)}{J_{n}^{2}\left(4 c t_{b} \cos \left(\delta_{y} b / 2\right) / e H v b\right)}
$$

The maximum of $\lambda$ at $\left(2 k_{\mathrm{F}}, 0,0\right)$ now drives, in increasing field, a first order transition from $\mathbf{Q}_{\mathbf{T}}$ to $\mathbf{Q}_{\mathbf{L}_{0}}$. In the high field phase, the critical temperature $T_{\mathrm{c}}\left(\delta_{x}, \delta_{v}\right)$ exhibits, as a function of the field, rapid oscillations. In fact, $\delta_{x}$ and $\delta_{y}$ vary with the field so that $T_{\mathrm{c}}(H)$ remains close to a relative maximum and jumps discontinuously from a maximum to the closer one, as happens in the transverse nesting case. Therefore, one expects a series of weakly first order transitions as the field is varied. In this scheme, the high field Shubnikov-de Haas like structure in the magnetoresistance is interpreted as signature of this series of transitions [20], with agreement between observed and predicted frequencies.

As in the transverse case, a Ginzburg-Landau calculation of the order parameter and of the free energy can be derived. We obtain the same expressions as in equations (3a) and (3b) where $t_{b}^{\prime}$ is replaced by $t_{b}$ and $b$ by $b / 2$. However, in this case the term $c_{3} f_{n}^{2}(H)$, which is negligible in the transverse case, becomes comparable with the other terms in the denominators of (3a) and (3b). Unfortunately, the approximations we have used until now in the evaluation of this term do not allow us to determine its sign with certainty, so that the slope of the transverse-longitudinal transition line within the SDW phases cannot be determined accurately. Furthermore, we cannot rule out that the coefficient $b_{n}$ in the longitudinal case becomes negative so that the metal-longitudinal SDW transition becomes first order and one needs the sixth order term in the LandauGinzburg expansion. This point, as well as the precise determination of the transition field deserves further work.

The threshold field $H_{0}$ below which the SDW is unstable, due to the finite width of the dispersion 
relation in the $c$-direction $[11,12]$ is obtained from Stoner's criterion at $T=0$. Assuming $t_{c_{i}} \ll t_{b_{i}}\left(t_{c_{i}}=t_{c}\right.$ or $t_{c}^{\prime}, t_{b_{i}}=t_{b}$ or $\left.t_{b}^{\prime}\right)$

$$
H_{0}\left(\mathbf{Q}_{i}\right)=\frac{2 \pi c t_{b_{i}}}{v e b_{i}} \operatorname{Ln}\left(\frac{\pi T_{0}\left(Q_{i}\right)}{2 \gamma t_{b_{i}}}\right) / \operatorname{Ln}\left(\frac{t_{c_{i}}}{t_{b_{i}}}\right) .
$$

In spite of the smaller interaction, the transverse threshold field is lower because of the larger anisotropy $t_{b}^{\prime} / t_{c}^{\prime}$ of the Fermi surface pocket. However, the wave vector $\mathbf{Q}_{3}=\left(2 k_{\mathrm{F}}, 0, \pi / c\right)$ yields a still larger anisotropy. The corresponding threshold field is obtained from (3) by setting $t_{b_{i}}=t_{b}$ and $t_{c_{i}}=t_{c^{\prime}}$. If $T_{0}\left(Q_{3}\right) \sim T_{0}\left(Q_{L_{0}}\right)$, then, it is possible, given the fact that the transition $T \rightarrow L_{0}$ occurs at $n_{\mathrm{T}}=1$, that the numerators in (4) are almost equal. In such a case, for that particular Q-dependence of the interaction, the denominators in (4) make $H_{0}\left(\mathbf{Q}_{3}\right)$ smaller. Therefore, it is not impossible that the lower threshold field is obtained in the $\mathbf{Q}_{3}$-regime. In such a case, one would obtain, with increasing field, three different nesting regimes : first the three-dimensional $\mathbf{Q}_{3}$ nesting, with low $H_{0}$, but also low ordering temperature, exhibiting a series of close transition, about 300 Oe apart, which would be difficult to resolve experimentally. Then as the field increases, the two-dimensional nesting $\mathbf{Q}_{\mathbf{T}}$ appears, and finally, the one-dimensional one $\mathbf{Q}_{\mathbf{L}_{0}}$. This particular case has been considered in figure 2 .

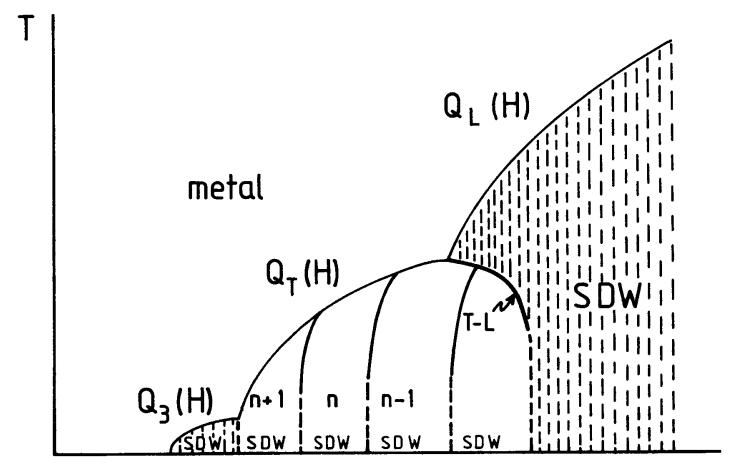

H

Fig. 2. - Phase diagram of the Field Induced SDW Phases in quasi-1D conductors. The transverse wave vector $\mathbf{Q}_{3}$ regime has been supposed to occur in low fields. It has many sub-phases (dotted lines) separated by magnetic field interval $\lesssim 0.03 \mathrm{~T}$. The phase in the intermediate field range has transverse nesting $Q_{\mathrm{T}}(H)$, with large component along $b$ so that the period from (quantized) sub-phase to sub-phase is $\sim 1 \times \mathrm{T}$ to $1.5 \mathrm{~T}$. The high field phase has longitudinal wave vector $Q_{\mathrm{L}}(H)$ so that again many sub-phases appear with small field interval $\lesssim 0.03 \mathrm{~T}$. The line T-L is strongly first order. No transition line is known accurately at low $T$. The slope of the T-L line may have the opposite sign.

The phase diagram sketched in figure 2 seems to account, at least qualitatively, for nearly all the available data. Let us mention that the model of longitudinal nesting in the whole field range proposed by Gor'kov and Lebed [11,20] does not seem consistent with the phase diagram inferred from the specific heat data [13]. Our theory gives rise to new experimental tests : for example, Shubnikov-de Haas like oscillations for $3.3 \mathrm{~T}<H<3.8 \mathrm{~T}$, discontinuous change of the SDW wave vector, etc... Fractional filling of Landau level is unlikely to be observed in the Bechgaard salts up to the largest fields available nowadays; as the field increases the transverse SDW phase with only one Landau level becomes unstable versus longitudinal nesting with many Landau levels.

Details on the microscopic Ginzburg-Landau calculation will appear in a forthcoming paper. 


\section{References}

[1] For a review see for example Jérome, D. and Schulz, H. J., Adv. Phys. 31 (1982) 299 and references therein.

[2] Kwak, J., Schirber, J., Greere, R. and Engler, E., Phys. Rev. Lett. 46 (1981) 1296.

[3] Garoche, P., Brusetti, R., Jérome, D. and BechgaArd, K., J. Physique Lett. 43 (1982) L-147.

[4] Kajimura, K., Tokumoto, H., Tokumoto, M., Murata, K., Anzai, H., Ishiguro, T. and Saito, G., J. Physique Colloq. 44 (1983) C3-1059.

[5] Ribault, M., Jérome, D., TuChendler, J., Weyl, C. and BechgaArd, K., J. Physique Lett. 44 (1983) L-953.

[6] Chaikin, P. M., Mu-Yong Choi, Kwak, J. F., Brooks, J. S., Martin, K. P., Naughton, M. J., Engler, E. M. and Greene, R. L., Phys. Rev. Lett. 51 (1983) 2333.

[7] Ribault, M., Cooper, J., Jérome, D., Mailly, D., Moradpour, A. and BechgaArd, K., J. Physique Lett. 45 (1984) L-935.

[8] Ribault, M., Mol. Cryst. Liq. Cryst. 119 (1985) 91.

[9] Azevedo, L., Schirber, J., Greene, R. and Engler, E., Physica B 108 (1981) 1183.

[10] Takahashi, T., Jérome, D. and BeChgaARD, K., J. Physique Lett. 43 (1982) L-565.

[11] GoR'Kov, L. P. and Lebed, A. G., J. Physique Lett. 45 (1984) L-433.

[12] Héritier, M., Montambaux, G. and Lederer, P., J. Physique Lett. 45 (1984) L-943.

[13] Chaikin, P. M. et al., Mol. Cryst. Liq. Cryst. 119 (1985) 79.

[14] Greene, R. L. and Chaikin, P. M., Physica B 126 (1984) 431.

[15] Garoche, P. and Pesty, F., to be published.

[16] Laughlin, R. B., Phys. Rev. Lett. 50 (1983) 1395.

[17] Thouless, D. J., Kohmoto, M., Nightingale, M. P. and Den Niss, M., Phys. Rev. Lett. 49 (1982) 405.

[18] Horowitz, B., Gutfreund, H., Weger, M., Solid State Commun. 39 (1981) 541.

[19] Héritier, M., Montambaux, G. and Lederer, P., Mol. Cryst. Liq. Cryst. 119 (1985) 97 ; see also YamaJI, K., Mol. Cryst. Liq. Cryst. 119 (1985) 105.

[20] GoR'Kov, L. P. and Lebed, A. G., Mol. Cryst. Liq. Cryst. 119 (1985) 73.

[21] Bando, H., Oshina, K., Suzuki, M., Kobayashi, H. and Saito, G., J. Phys. Soc. Japan 51 (1982) 2711.

[22] Ulmet, J. P., Auban, P. and Askenazy, S., Solid State Commun. 52 (1984) 567.

[23] Toffano, Z., Roger, M. and Delrieu, J. M., to be published.

[24] BARISIC, S., private communication.

[25] GoR'Kov, L. P., J.E.T.P. 9 (1959) 1364. 